\title{
Weak cosmic censorship with self-interacting scalar and bound on charge to mass ratio
}

\author{
Yan Song, Tong-Tong $\mathrm{Hu}$ and Yong-Qiang Wang ${ }^{1}$ \\ Research Center of Gravitation 8 Institute of Theoretical Physics $\&$ \\ Key Laboratory for Magnetism and Magnetic of the Ministry of Education, Lanzhou University, \\ Lanzhou 730000, China \\ E-mail: songy18@lzu.edu.cn, hutt17@lzu.edu.cn, yqwang@lzu.edu.cn
}

ABSTRACT: We study the model of four-dimensional Einstein-Maxwell- $\Lambda$ theory minimally coupled to a massive charged self-interacting scalar field, parameterized by the quartic and hexic couplings, labelled by $\lambda$ and $\beta$, respectively. In the absence of scalar field, there is a class of counterexamples to cosmic censorship. Moreover, we investigate the full nonlinear solution with nonzero scalar field included, and argue that these counterexamples can be removed by assuming charged self-interacting scalar field with sufficiently large charge not lower than a certain bound. In particular, this bound on charge required to preserve cosmic censorship is no longer precisely the weak gravity bound for the free scalar theory. For the quartic coupling, for $\lambda<0$ the bound is below the one for the free scalar fields, whereas for $\lambda>0$ it is above. Meanwhile, for the hexic coupling the bound is always above the one for the free scalar fields, irrespective of the sign of $\beta$.

KEYwords: Black Holes, Spacetime Singularities

ARXIV EPRINT: 2008.02513

\footnotetext{
${ }^{1}$ Corresponding author.
} 


\section{Contents}

1 Introduction 1

2 The model and ansatz 2

3 Numerical results $\quad 4$

3.1 Linear solutions 5

$\begin{array}{lll}3.2 & \text { Full nonlinear solutions } & 6\end{array}$

3.2.1 Solutions with $\lambda \neq 0$ and $\beta=0 \quad 6$

3.2.2 Solutions with $\lambda=0$ and $\beta \neq 0 \quad 8$

4 Conclusions $\quad 11$

\section{Introduction}

The weak cosmic censorship conjecture (WCCC), first put forward by Penrose in 1969 [1], states that there is no naked singularity, a region of arbitrarily large curvature visible to distant observers, except the cosmological singularities, and the singularity that arises in the gravitational collapse $[2,3]$ must be hidden behind event horizon, never to be observed by external observers. In past several decades, based on Wald's gedanken experiment, many significant works have been done to check this conjecture. Some of them have indeed proved its validity under certain conditions [4-21], while the others attempt to present its violations [22-26]. Besides, other various possible examples to violate cosmic censorship are also proposed. For instance, a naked singularity can arise from smooth initial data due to the Gregory-Laflamme instability [27] of high dimensional black objects, which can cause the horizon unstable to pinch off in finite time, leading to violation of the cosmic censorship [28-32]. In addition, in four dimensions, a promising class of counterexamples $[33,34]$ has been conjectured in Einstein-Maxwell theory with negative cosmological constant, and not involving Maxwell field, the authors also constructed a class of vacuum counterexamples with differential rotation boundary in [35].

Recently, the authors [36] proposed that this class of counterexamples presented in [33] can be removed by assuming the weak gravity conjecture (WGC) [37-40], which tells whether or not a low-energy effective theory is consistent with quantum theory and ensures that gravity is the weakest force. As discussed in [36], adding massive charged scalar field with sufficiently large charge, the original Einstein-Maxwell solution in AdS will become unstable and then settle down to a new stationary solution with scalar hair, the dual analogy of holographic superconductor [41-43], for which the cosmic censorship is no longer violated in the previous way. In particular, the minimum charge-to-mass ratio required to preserve cosmic censorship is precisely the bound predicted by the weak gravity conjecture. 
It asserts that if the weak gravity conjecture holds, the weak cosmic censorship can be preserved. For more evidences, see [44]. Moreover, based on Born-Infeld electrodynamics coupling to gravity [45], the authors also constructed analogous counterexamples to cosmic censorship and found that these counterexamples can still be removed by including charged scalar field with sufficiently charge.

In this work, we investigate a generalisation of the scenario proposed in [36] by including quartic and hexic couplings, which have been investigated a lot in the literature [46-51]. One might wonder what happens to the bound on charge required to preserve cosmic censorship, if we consider the self-interacting scalar field, rather than the non-self-interacting one. To answer this, in this work, we follow the procedure in [36] and calculate the bounds for various quatic and hexic couplings. Besides, we note that, compared with other possible potential terms about complex scalar fields, as shown in [52], our major motivation for studying quartic and hexic couplings stems from two considerations. First, these generic potential terms, such as Cosh-Gordon potential, always act as the attractive or repulsive force, and these attractive and repulsive interactions can be investigated in the study for quartic and hexic terms, through the choice of coupling parameters. Second, when the scalar field is small, the fourth and sixth order terms in series expansion of these generic potentials are respectively the quartic and hexic terms we study, and thus for these we can expect a qualitatively similar result to that for quartic and hexic couplings. Notice that for large scalar fields, the generic potentials are manifestly different from the quartic and hexic couplings.

The paper is organised as follows. In section 2, we construct Einstein-Maxwell-scalar model with negative cosmological constant, in consideration of the quartic and hexic selfinteractions. In section 3, we analysis the stability of solutions without charged fields and then numerically investigate the full nonlinear solutions for the quartic coupling and the hexic coupling, respectively, to give the bound on charge required to preserve cosmic censorship. Finial, we conclude our results in section 4 .

\section{The model and ansatz}

To remove the counterexamples proposed in [33], we add massive charged self-interacting scalar field $\Phi$ into the original Einstein-Maxwell gravity. The resulting action $\mathcal{L}$ is given by

$$
S=\frac{1}{16 \pi G} \int d^{4} x \sqrt{-g}\left[R+\frac{6}{L^{2}}-F^{a b} F_{a b}+\mathcal{L}_{\text {scalar }}\right],
$$

where $L$ is the AdS length scale for which we take $L=1$, and $G$ the gravitational constant. Moreover, $F_{a b}$ is the electromagnetic strength tensor with respect to $A$, the $\mathrm{U}(1)$ gauge potential, by $F \equiv d A$. The self-interacting scalar term $\mathcal{L}_{\text {scalar }}$ reads

$$
\begin{aligned}
\mathcal{L}_{\text {scalar }} & =-4\left(\mathcal{D}_{a} \Phi\right)\left(\mathcal{D}^{a} \Phi\right)^{\dagger}-4 U(|\Phi|) \\
\text { with } \quad U(|\Phi|) & =m^{2} \Phi \Phi^{\dagger}+\lambda\left(\Phi \Phi^{\dagger}\right)^{2}+\beta\left(\Phi \Phi^{\dagger}\right)^{3},
\end{aligned}
$$

where $\mathcal{D}=\nabla-i q A$ is the covariant derivative with respect to $A$. The scalar field $\Phi$ is of mass $m$ and charge $q$, having quartic and hexic couplings, labelled by $\lambda$ and $\beta$, respectively. 
The free scalar field has $\lambda=\beta=0$. We note that in anti-de Sitter spacetime, the mass squared of scalar field we take must is at or above a lower bound to ensure a stable AdS spacetime, in which this bound is the Breitenlohner-Freedman (BF) bound [53-56]. For $(3+1)$-dimensions, we therefore take $m^{2} \geq-9 / 4$.

From eqs. (2.1), the equations of motion for this model can be derived as

$$
\begin{aligned}
R_{a b}+\frac{3}{L^{2}} g_{a b}= & 2\left(F_{a}^{c} F_{b c}-\frac{1}{4} g_{a b} F^{c d} F_{c d}\right)+2\left(\mathcal{D}_{a} \Phi\right)\left(\mathcal{D}_{b} \Phi\right)^{\dagger}+2\left(\mathcal{D}_{a} \Phi\right)^{\dagger}\left(\mathcal{D}_{b} \Phi\right) \\
& +2 m^{2} g_{a b} \Phi \Phi^{\dagger}+2 \lambda g_{a b}\left(\Phi \Phi^{\dagger}\right)^{2}+2 \beta g_{a b}\left(\Phi \Phi^{\dagger}\right)^{3} \\
\nabla_{a} F_{b}^{a}= & i q\left[\left(\mathcal{D}_{b} \Phi\right) \Phi^{\dagger}-\left(\mathcal{D}_{b} \Phi\right)^{\dagger} \Phi\right] \\
\mathcal{D}_{a} \mathcal{D}^{a} \Phi= & m^{2} \Phi+2 \lambda\left(\Phi \Phi^{\dagger}\right) \Phi+3 \beta\left(\Phi \Phi^{\dagger}\right)^{2} \Phi
\end{aligned}
$$

In the special case $\Phi=0$, this model reduces to the previous Einstein-Maxwell theory in AdS which is konwn to have a class of counterexamples to cosmic censorship [33]. However, when considering $\Phi \neq 0$, the situation is complicated and we need to numerically solve eqs. (2.2). Before this we need a set of appropriate ansatz and boundary conditions for full nonlinear solutions with charged scalar.

In this work, we focus on static, axisymmetric metric, which indicates that our coordinate system can be chosen such that $\partial_{t}$ and $\partial_{\phi}$ are Killing fields. A simplest configuration of this form is given by the pure AdS solution in Poincaré coordinates

$$
d s^{2}=\frac{L^{2}}{z^{2}}\left[-d t^{2}+d r^{2}+r^{2} d \phi^{2}+d z^{2}\right] .
$$

Note that in Cartesian coordinates $(r, z)$, both $r$ and $z$ are range of values in $[0, \infty)$. We therefore simply perform a Cartesian to polar-like transformation in the following way,

$$
z=\frac{y \sqrt{2-y^{2}}}{1-y^{2}}\left(1-x^{2}\right), \quad r=\frac{y \sqrt{2-y^{2}}}{1-y^{2}} x \sqrt{2-x^{2}} .
$$

This allows us to integrate over a finite domain $[0,1] \times[0,1]$.

In order to obtain more general solutions, we give appropriate ansatz for metric and matter in terms of the indefinite functions $Q_{i}$, with $i=1,2, \ldots, 7$, as functions of coordinates $x$ and $y$. The most general metric ansatz for static and axisymmetric solutions is given by

$$
\begin{aligned}
d s^{2}= & \frac{L^{2}}{\left(1-x^{2}\right)^{2}}\left[-\frac{\left(1-y^{2}\right)^{2} Q_{1} d t^{2}}{y^{2}\left(2-y^{2}\right)}+\frac{4 Q_{4}}{2-x^{2}}\left(d x+\frac{Q_{3}}{1-y^{2}} d y\right)^{2}\right. \\
& \left.+\frac{4 Q_{2} d y^{2}}{y^{2}\left(1-y^{2}\right)^{2}\left(2-y^{2}\right)^{2}}+x^{2}\left(2-x^{2}\right) Q_{5} d \phi^{2}\right]
\end{aligned}
$$

and for matter fields we take

$$
A=L Q_{6} d t, \quad \text { and } \quad \Phi=\left(1-x^{2}\right)^{\triangle} y^{\triangle}\left(2-y^{2}\right)^{\frac{\triangle}{2}} Q_{7},
$$

with $\triangle \equiv 3 / 2+\sqrt{9 / 4+L^{2} m^{2}}$, playing the role of mass in AdS. Here we take $\triangle=2$, corresponding to $m^{2}=-2$. 
In our analysis, we choice the same boundary conditions as those presented in [36]. Notice that, in the absence of scalar field, in [57] the authors showed that for different asymptotic profiles of vector potential, the solutions of the Einstein-Maxwell theory in AdS always appear to be singular as long as these profiles fall off faster than $1 / r$ at large $r$. This result does not depend on a quite artificial model. So, we also adopt the asymptotic profile decaying faster than $1 / r$ studied in [57], and transform it into polar-like coordinates. At $x=1$, corresponding to the conformal boundary, we demand the asymptotic metric to be flat, which imposes

$$
Q_{1}=Q_{2}=Q_{4}=Q_{5}=1, \quad Q_{3}=0, \quad Q_{6}=a\left(1-y^{2}\right)^{n}, \quad \text { and } \quad \frac{\partial Q_{7}}{\partial x}=0,
$$

where $a$ is an amplitude and $n$ denotes the fall-off behavior of asymptotic potential for which we demand $n>2$. At $x=0$, corresponding to the symmetry axis, regularity demands

$$
\frac{\partial Q_{1}}{\partial x}=\frac{\partial Q_{2}}{\partial x}=\frac{\partial Q_{4}}{\partial x}=\frac{\partial Q_{5}}{\partial x}=\frac{\partial Q_{6}}{\partial x}=\frac{\partial Q_{7}}{\partial x}=0, \quad Q_{3}=0, \quad \text { and } \quad Q_{4}=Q_{5} .
$$

At the intersection of the conformal boundary with the axis of symmetry located at $r=z=0$, corresponding to the point $y=0$, we impose

$$
Q_{1}=Q_{2}=Q_{4}=Q_{5}=1, \quad Q_{3}=0, \quad Q_{6}=a, \quad \text { and } \quad \frac{\partial Q_{7}}{\partial y}=0 .
$$

At the Poincare horizon for $n>1$, located at $y=1$, we impose

$$
Q_{1}=Q_{2}=Q_{4}=Q_{5}=1, \quad \text { and } \quad Q_{3}=Q_{6}=Q_{7}=0 .
$$

Given ansatz and boundary conditions, we could search for the full nonlinear solutions to eqs. (2.2) numerically and further investigate their properties, as we will discuss below.

\section{Numerical results}

We use the Deturck method [58] to numerically solve the equations of motion eqs. (2.2) with appropriate boundary conditions, and to present the stationary smooth solutions of our model. The Deturck method, first introduced in [58] and reviewed more in [59], is a effective tool to solve the complex Einstein equation numerically. Following the same strategy as in [59], one can deform eq. (2.2a) by adding a gauge fixing term and construct a set of solvable elliptic equations, the so-called Einstein-Deturck equation system, which reads

$$
\begin{aligned}
R_{a b}+\frac{3}{L^{2}} g_{a b}-\nabla_{(a} \xi_{b)}= & 2\left(F_{a}^{c} F_{b c}-\frac{1}{4} g_{a b} F^{c d} F_{c d}\right)+2\left(D_{a} \Phi\right)\left(D_{b} \Phi\right)^{\dagger}+2\left(D_{a} \Phi\right)^{\dagger}\left(D_{b} \Phi\right) \\
& +2 m^{2} g_{a b} \Phi \Phi^{\dagger}+2 \lambda g_{a b}\left(\Phi \Phi^{\dagger}\right)^{2}+2 \beta g_{a b}\left(\Phi \Phi^{\dagger}\right)^{3}
\end{aligned}
$$

with $\xi^{a}=\left[\Gamma_{c d}^{a}(g)-\Gamma_{c d}^{a}(\bar{g})\right] g^{c d}$. Notice that, $\Gamma_{c d}^{a}(\bar{g})$ is the Levi-Civitta connection with respect to the reference metric $\bar{g}$, which is introduced to fix the residual degree of gauge freedom for the metric $g$ we intend to determine. For the reference metric, we take $Q_{1}=Q_{2}=Q_{4}=Q_{5}=1$ and $Q_{3}=0$. 

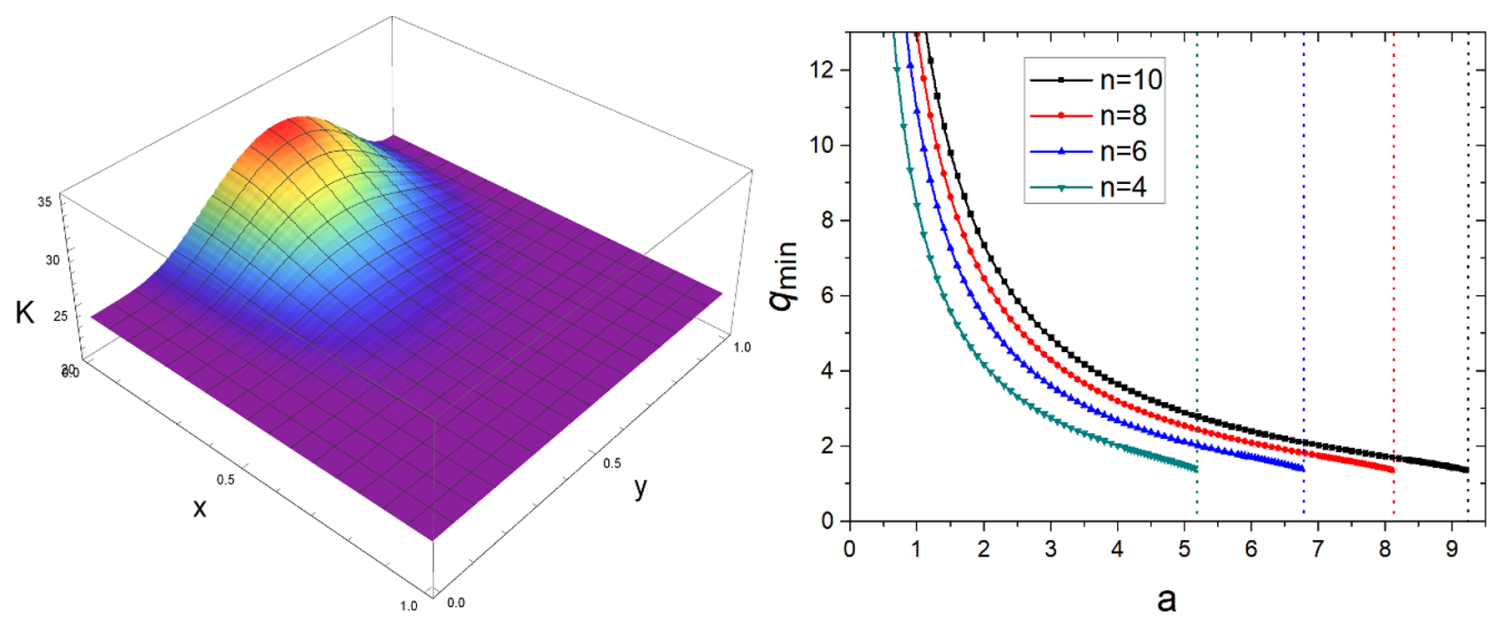

Figure 1. Left: the distribution of the Kretschmann scalar $K$ over the integrate domain $[0,1] \times[0,1]$, for $a=5$ and $n=8$. Right: the minimal charge $q_{\min }$ against amplitude $a$ with fixed $\lambda=4$ and $\beta=6$, for various profiles $n=10,8,6,4$.

Furthermore, we numerically solve the elliptic equations using the finite element method over the integrate domain $[0,1] \times[0,1]$, and the iterate approach is Newton-Raphson methods. In particular, employing non-uniform grids allows us to refine some high gradient regions which become more important as we approach singular solutions, such that desired accuracy can be reached in smaller resource. Note that to ensure our results correct, in this work, $200 \times 300$ grid is used and the relative error below $10^{-5}$ is required.

\subsection{Linear solutions}

In four-dimensional Einstein-Maxwell theory in AdS, as amplitude $a$ increases, the electric field and spacetime curvature grow as well [33], gradually away from the pure AdS geometry. To investigate the change of spacetime curvature, one can evaluate a curvature invariant. We here calculate the Kretschmann scalar, $K=R_{\alpha \beta \gamma \delta} R^{\alpha \beta \gamma \delta}$, which is constructed by the square of Reimann curvature tensor $R_{\alpha \beta \gamma \delta}$.

We review the distribution of scalar $K$ in [33] in $x-y$ plane for $n=8$ and $a=5$, as seen in the left panel of figure 1 . The scalar $K$ is shown to develop a maximum value at the boundary $x=0$, corresponding to the axis of symmetry. However, away from this axis the change in amplitude does not dramatically affect the spacetime curvature and $K$ appears to remain that for pure AdS spacetime, $K=24 / L^{4}$, as shown by the purple region.

To see if there are solutions with nonzero scalar field included, a simple strategy is to first investigate the stability of the original Einstein-Maxwell solution [36]. In general, the onset of instabilities can lead to new stationary solutions. According to the linear perturbation theory [59], we consider a perturbative self-interacting scalar field around a fixed Einstein-Maxwell background, and then the field equation eq. (2.2c) reduces to a linear equation

$$
\left(\nabla_{a} \nabla^{a}-m^{2}\right) \Phi-2 \lambda\left(\Phi \Phi^{\dagger}\right) \Phi-3 \beta\left(\Phi \Phi^{\dagger}\right)^{2} \Phi=q^{2} A_{a} A^{a} \Phi
$$


Note that in the probe limit, the scalar field along the zero-mode curve is so small that its higher order nonlinear terms are negligible. Hence, perturbative equation eq. (3.2) also reads

$$
\left(\nabla_{a} \nabla^{a}-m^{2}\right) \Phi=q^{2} A_{a} A^{a} \Phi
$$

which is just the free scalar perturbative equation studied in [36]. This indicates that our perturbative results need to be irrelevant to self-interacting couplings.

The numerical results for zero-modes of linear perturbations eq. (3.2) are presented in the right panel of figure 1 , where we plot the minimal charge $q_{\min }$ against amplitude $a$ for several profiles $n=10,8,6,4$. For fixed $n$ profile, the curve always ends at a maximum amplitude $a=a_{\max }$, which is marked by the vertical dotted line. Once slightly above the maximum amplitude $a_{\max }$, the electric field will diverge and spacetime curvature grows without bound, indicating that a class of counterexamples to weak cosmic censorship appears. As expected, these results are completely the same as those for $\lambda=\beta=0$.

Notice that, above this zero-mode curve, the original Einstein-Maxwell solutions will become unstable to condense charged scalar field, and then settle down to new stationary hairy solutions. Thus, to see whether these counterexamples to weak cosmic censorship can be removed with charged self-interacting scalar field, we investigate the full nonlinear solutions derived from eqs. (2.2) for the quartic coupling $\lambda \neq 0$ and the hexic coupling $\beta \neq 0$, respectively.

\section{$3.2 \quad$ Full nonlinear solutions}

\subsubsection{Solutions with $\lambda \neq 0$ and $\beta=0$}

To investigate the existence of solutions with $\Phi \neq 0$, we monitor the condensation of scalar field $\Phi$ defined in eqs. (2.5b) by computing the expectation value of its holographic dual boundary operator $\mathcal{O}_{2},\left\langle\mathcal{O}_{2}\right\rangle=\left(1-y^{2}\right)^{2} Q_{7}$, according to AdS/CFT duality. Note that we take $n=8$ and $q=2.15>q_{\min }$ to ensure scalar condensation, where $q_{\min }$ is the minimal charge deduced in stability analysis.

In figure 2, the left panel shows the distribution of scalar condensate $\left\langle\mathcal{O}_{2}\right\rangle$ at conformal boundary with fixed $a=9$ for various couplings $\lambda=-1,0,2$, represented by green, black and red solid lines, respectively. We show that for a given value of $r$, as $\lambda$ decreases, the scalar condensate $\left\langle\mathcal{O}_{2}\right\rangle$ increases. Meanwhile, the right panel shows the distribution of scalar condensate $\left\langle\mathcal{O}_{2}\right\rangle$ with fixed $\lambda=1$. From top to bottom, these are for different $a=10,9,8,5.9$. For a given value of $r$, the scalar condensate $\left\langle\mathcal{O}_{2}\right\rangle$ increases with increasing $a$. In addition, from figure 2, the scalar condensate is always maximized at the origin $r=0$ and decays to zero at infinity. This is in agreement with the feature of holographic dual superconductor.

From above, for fixed $q=2.15$, there indeed exist smooth solutions with quartic hair for large amplitudes $a$, and the condensate is more likely to form for larger $a$. This indicates that the hairy solutions can exist for arbitrarily large $a$, never to be singular.

To check this, in the left panel of figure 3 , we present the central value of scalar condensate, $\left\langle\mathcal{O}_{2}\right\rangle_{r=0}$, at origin $r=0$ against amplitude $a$ with fixed $n=8$ and $q=2.15$. These curves are for $\lambda=-1$ (green), $\lambda=0$ (black) and $\lambda=2$ (red), respectively. For 

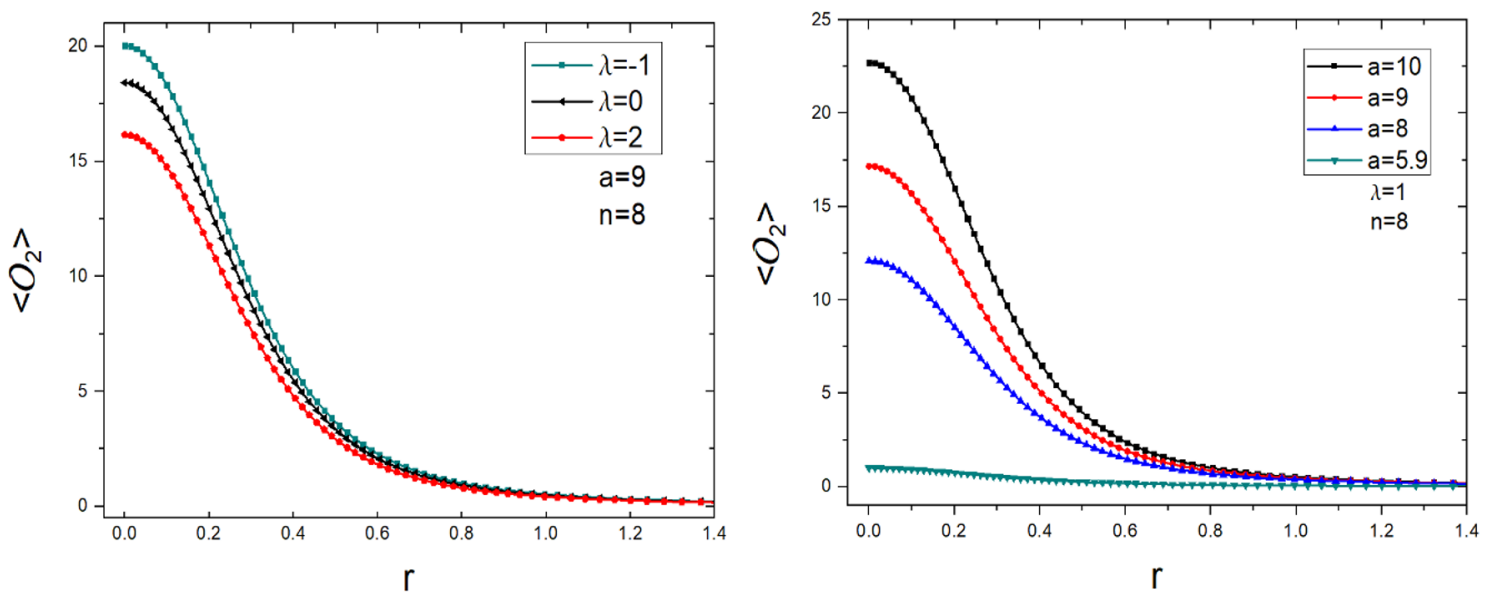

Figure 2. Left: $\left\langle\mathcal{O}_{2}\right\rangle$ against $r$, for several $\lambda=-1,0,2$. The black solid line is the non-selfinteracting result for reference. Right: $\left\langle\mathcal{O}_{2}\right\rangle$ against $r$. From top to bottom, we have $a=10,9,8,5.9$. For both panels we take $n=8$ and $q=2.15$.
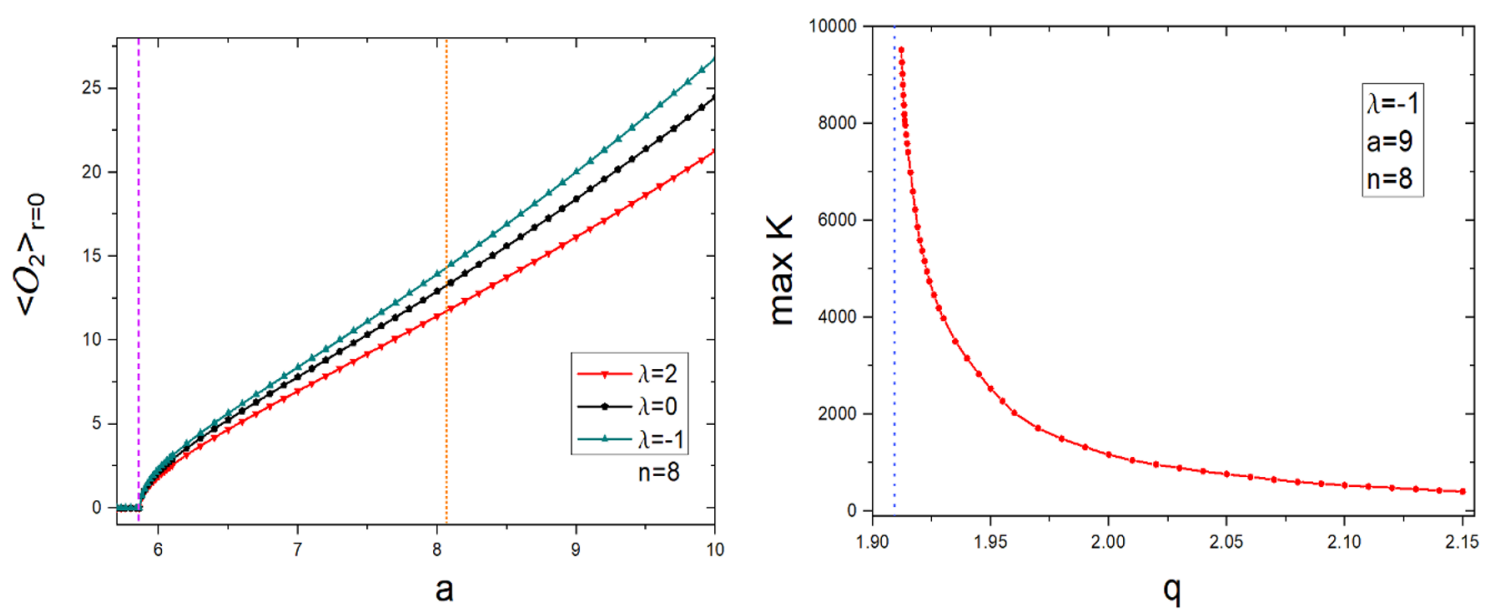

Figure 3. Left: scalar condensate $\left\langle\mathcal{O}_{2}\right\rangle$ at $r=0$ against $a$ at fixed $q=2.15$ for $\lambda=-1,0,2$. The purple dashed vertical gridline corresponds to $a=5.86$ while the orange dotted one to $a_{\max }=8.06$. Right: the maximum $K$ over spacetime against scalar charge $q$, computed with $\lambda=-1$ and $a=9$. The vertical gridline marks $q_{\min }=1.909$. Both panels take $n=8$.

each value of $\lambda$, the scalar condensate $\left\langle\mathcal{O}_{2}\right\rangle$ always starts to form around $a=5.86$, marked by the purple dashed vertical gridline, and then monotonously increases with increasing $a$ even for large amplitude $a>a_{\max }$, where $a_{\max }$, marked by orange dotted vertical gridline, is the maximum amplitude for smooth Einstein-Maxwell solutions. It seems that the hairy solutions can exist for arbitrarily large $a$. This means that we have removed the previous counterexamples to weak cosmic censorship for $q=2.15$ and $n=8$.

The right panel of figure 3 is a plot of the maximum value of Kretschmann scalar $K$ as a function of scalar charge $q$, with the vertical dotted gridline corresponding to $q_{\text {min }}=1.909$. We set $\lambda=-1$ and $a=9$, and slowly decrease $q$ until meet an obstruction at 


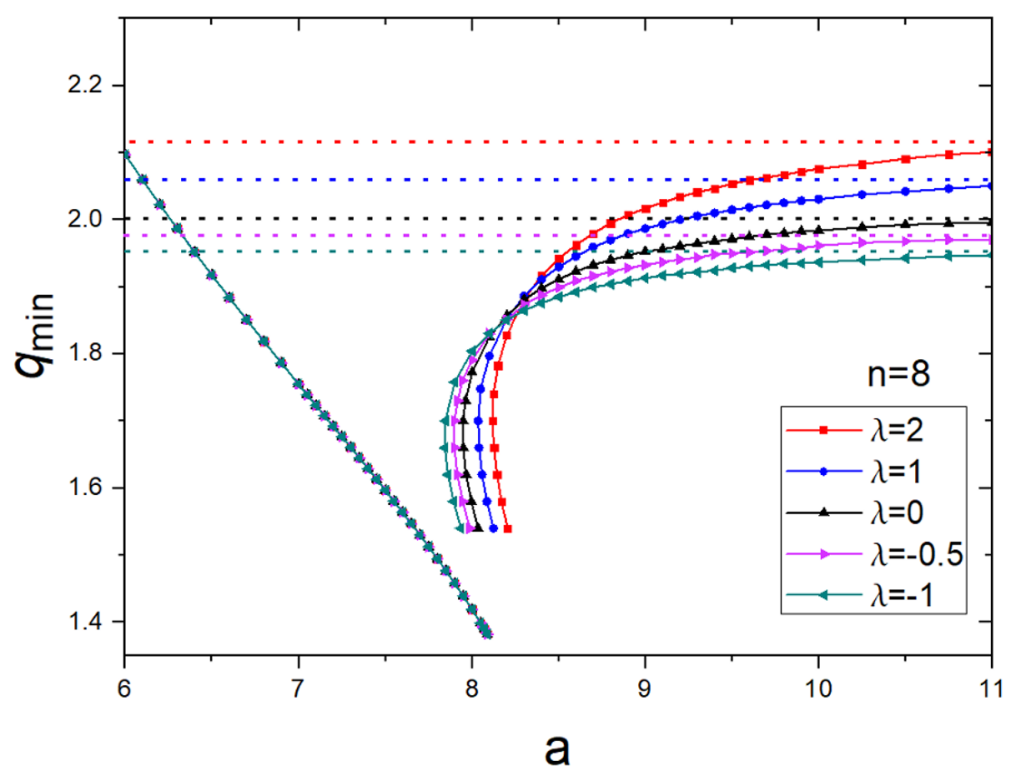

Figure 4. Phase diagram of solutions with the minimal charge $q_{\min }$ as a function of the amplitude $a$ with profile $n=8$, for several values of coupling parameter $\lambda$.

$q=q_{\min }$. The blow up at $q<q_{\min }$ indicates the appearance of a singularity. This implies that weak cosmic censorship can still be violated for large amplitude at small charge, and we therefore require sufficiently large scalar field charge $q$ to preserve cosmic censorship. Furthermore, a important question is what is the bound on charge required to preserve WCCC for arbitrarily large $a$. We will discuss this in what follows.

In figure 4 we compute the minimum charge $q_{\min }$ versus various amplitude $a$ with fixed $n=8$ profile. These red, blue, purple and green solid lines correspond to $\lambda=2,1,-0.5,-1$, respectively, with the respective asymptotes located at $q_{\min }=2.12,2.06,1.975,1.95$. The black solid line is the non-self-interacting result for reference. For each value of $\lambda$, there always exist two unconnected curves, one of which plotted for $a<a_{\max }$ is just the onset of scalar condensate shown in figure 1 , and the other for larger $a$ is the singular curve.

For each $\lambda \neq 0$, singular curve asymptotically approaches a certain bound on charge, as seen in figure 4. This is consistent with the result for $\lambda=0$. Moreover, the presence of self-interaction also leads to manifest differences. We show that the bound on charge presented in figure 4 would decrease with decreasing $\lambda$, instead of remaining the one for free scalar fields $q=2$. In particular, for solutions with $\lambda>0$, the bounds are located at $q>2$, but for those with $\lambda<0$ they appear at $q<2$. That is to say, if we take $\lambda<0$, the violation would not occur even through we take $q$ slightly below $q=2$, which is not possible in the free scalar case.

\subsubsection{Solutions with $\lambda=0$ and $\beta \neq 0$}

To further investigate the implication of self-interaction potential, one can also analysis the full nonlinear solutions with hexic hair. In figure 5 the distribution of scalar condensate at conformal boundary is computed with $n=8$ and $q=2.1$. From top to bottom, these 

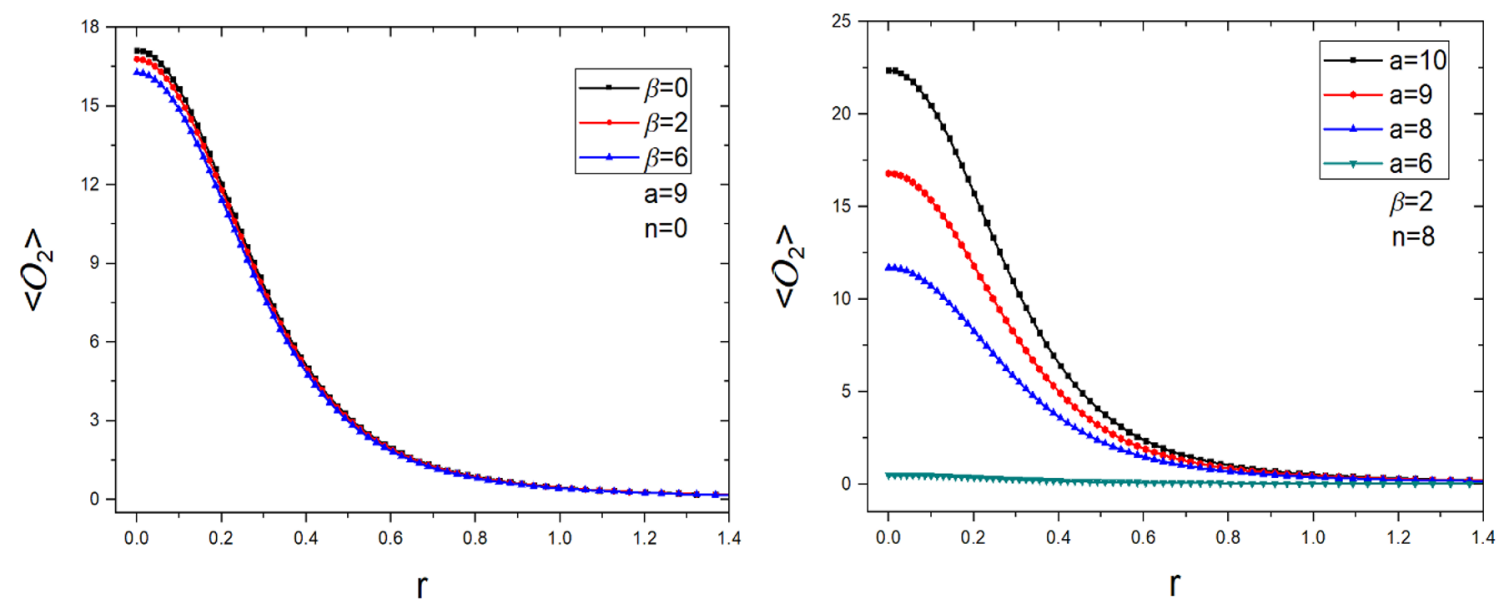

Figure 5. $\left\langle\mathcal{O}_{2}\right\rangle$ as a function of $r$, for several values of $\beta$ (left) and a (right).
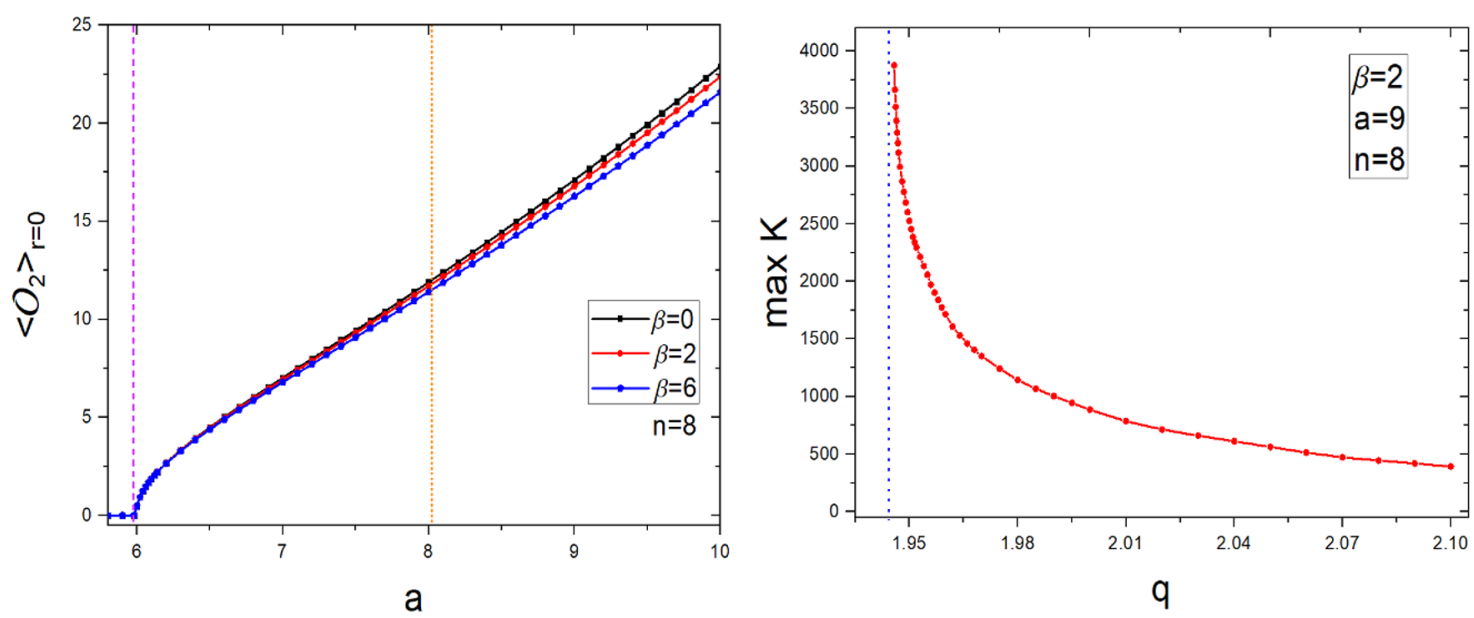

Figure 6. Left: $\left\langle\mathcal{O}_{2}\right\rangle_{r=0}$ against $a$ at fixed $q=2.1$, for several values of $\beta>0$. The purple dashed vertical gridline corresponds to $a=5.98$ while the orange dotted one to $a_{\max }=8.06$. Right: approach to the singular solution for fixed $\beta=2$ and $a=9$. The vertical gridline marks $q_{\min }=1.945$.

are for various $\beta=0,2,6$ (left) and for various $a=10,9,8,6$ (right), respectively. Similar to the results presented in figure 2 , the maximum condensate is always reached at origin $r=0$. One can see that for $\beta>0$, the scalar condensate $\left\langle\mathcal{O}_{2}\right\rangle$ decreases as $\beta$ increases, and increases as $a$ increases. This implies that for $q=2.1$, there indeed exist smooth stationary solutions with hexic hair for large amplitude $a$ and the hairy solutions could exist for arbitrarily large $a$.

To check this, the central value of scalar condensate $\left\langle\mathcal{O}_{2}\right\rangle_{r=0}$ is computed with $q=2.1$, as a function of $a$. The numerical results are plotted in the left panel of figure 6 , where these curves are for various $\beta=0,2,6$, represented by the black, red and blue solid lines, respectively. We also mark both the onset of scalar condensate, $a=5.98$, and $a_{\max }=8.06$. For all cases for fixed $q=2.1$, the condensation curves always start with around $a=5.98$ 


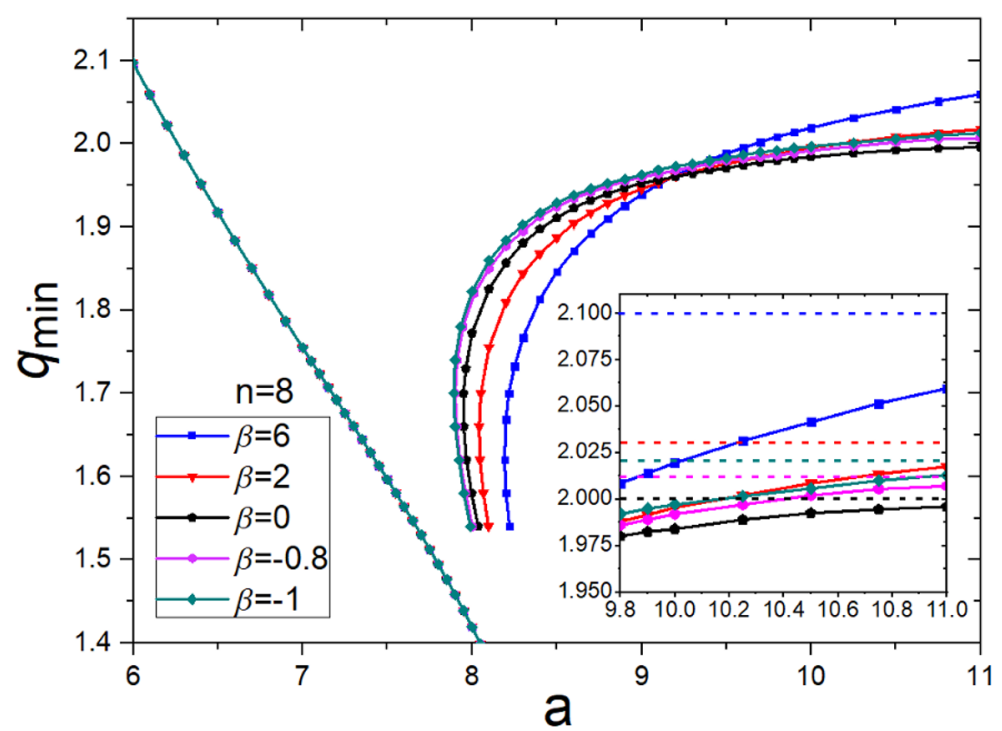

Figure 7. Phase diagram of the minimal charge $q_{\min }$ as a function of the amplitude $a$, with fixed $n=8$ and for several values of $\beta=6,2,-0.8,-1$, and the black solid line represents $\beta=0$ for reference.

and monotonously increase with increasing $a$, likely to extend for arbitrarily large $a$. This implies that we have removed the previous counterexamples to cosmic censorship for $q=2.1$, in the presence of hexic hair.

However, we again find that there are counterexamples to weak cosmic censorship for large amplitudes even including the scalar field in the hexic case, following in the quartic case. As can be observed in the right panel of figure 6 , we take for instance $\beta=2$ and $a=9$, and plot the maximum of the Kretschmann scalar $K$ as a function of charge $q$. It is clear that the maximum $K$ appears to grow without bound as we lower the charge below a minimum $q_{\min }=1.945$, denoted by the vertical gridline, which indicates the appearance of a singularity. This is to say, to avoid this singularity the sufficiently large scalar field charge is necessary.

One may wonder if there are also the lower bounds on charge required to preserve WCCC in the hexic case, as shown in the quartic case. In order to answer this, we have computed $q_{\text {min }}$ versus various $a$ in figure 7 . More details are shown in the inset. These blue, red, purple and green solid lines show $\beta=6,2,-0.8,-1$, respectively, with the black solid line for $\beta=0$ for reference. The horizonal dotted gridlines are respectively the asymptotes of these singular curves, corresponding to $q_{\min }=2.10,2.03,2.012,2.02$. It is clear that for each $\beta$, the singularities in hexic solutions still approach a certain bound, and the value of bound increases with the increasing absolute value of hexic coupling $\beta$. That is, for both $\beta>0$ and $\beta<0$, the bounds are always located at $q>2$, above the one for the free scalar fields. 


\section{Conclusions}

In this work, we include massive charged quartic and hexic scalar fields to the original Einstein-Maxwell theory in AdS, which is known to have a class of counterexamples to cosmic censorship. Similar to the non-self-interacting case studied in [36], we find that if we require the scalar field charge not lower than a certain bound, then these counterexamples in four dimensional AdS spacetime can be removed. Besides, the self-interaction, despite does not affect the onset curve at $a<a_{\max }$, indeed manifestly modifies the singular curves at larger $a$. The bound for self-interacting theory is no longer precisely the weak gravity bound for free scalar theory. For the quartic coupling, for $\lambda<0$ the new bound is below the one for the free scalar fields, whereas for $\lambda>0$ it is above. Meanwhile, for the hexic coupling the bound is always above the one for the free scalar fields, irrespective of the sign of $\beta$. Note that we take for instance $n=8$, but for different values of $n>2$, our results about the bound on charge are generic and do not depend on a quite artificial model.

In the free scalar case, the weak gravity bound is precisely the minimum charge required to preserve cosmic censorship, implying the possible connection between WGC and WCCC. That is the main idea of [36]. We need to note that, though for $\lambda<0$ the bounds on charge are always below the one for the free scalar fields, this does not imply that the weak gravity-cosmic censorship connection is invalid in self-interacting theory. It is more likely that the weak gravity bound is modified due to the presence of self-interaction. Once confirmed that the modified weak gravity bound is again precisely what is required to preserve cosmic censorship we obtained, we can give more information on this possible connection between these two conjectures.

In this work, we have shown that these counterexamples can be removed by including charged self-interacting scalar field with sufficiently large charge. However, up to now we have not done the complete time-dependent evolution (analogous to [34]). In [34], the authors showed that a class of time-dependent solutions evolves to be singular in the complete time-dependent evolution, and thus provided the first counterexample to the weak cosmic censorship conjecture in four dimensional Einstein-Maxwell theory with negative cosmological constant. Therefore, a meaningful work is to investigate what happens in the complete time-dependent evolution when massive charged scalar field is included. To do this, one can follow the procedure described in [34], and ask whether the time-dependent solutions with nonzero scalar field included can still evolve to be singular over time, for different values of charge-to-mass ratio. This is an obvious avenue of research that deserves further attention.

Besides, we can also give a comment about the multi-charged case. In this work, we have focused mostly on the case of a single Maxwell field, but from [44], one can also construct a similar model of two or more Maxwell fields coupled to gravity and find similar counterexamples to cosmic censorship. Therefore, two natural problems are whether these counterexamples can be removed when we include two or more massive charged selfinteracting scalar fields with different charges in the original solutions without charged scalars, and whether the minimal charge required to preserve the cosmic censorship is precisely the weak gravity bound if it exists. We will discuss these in the future work. 


\section{Acknowledgments}

We thank Shuo Sun and Hong-Bo Li for discussions. Some computations were performed on the shared memory system at institute of computational physics and complex systems in Lanzhou university. This work was supported by the Fundamental Research Funds for the Central Universities (Grants No. lzujbky-2017-182).

Open Access. This article is distributed under the terms of the Creative Commons Attribution License (CC-BY 4.0), which permits any use, distribution and reproduction in any medium, provided the original author(s) and source are credited.

\section{References}

[1] R. Penrose, Gravitational collapse: The role of general relativity, Riv. Nuovo Cim. 1 (1969) 252 [INSPIRE].

[2] S.W. Hawking, Breakdown of Predictability in Gravitational Collapse, Phys. Rev. D 14 (1976) 2460 [InSPIRE].

[3] P.S. Joshi and D. Malafarina, Recent developments in gravitational collapse and spacetime singularities, Int. J. Mod. Phys. D 20 (2011) 2641 [arXiv:1201.3660] [INSPIRE].

[4] R.M. Wald, Gedanken experiments to destroy a black hole, Ann. Phys 82 (1974) 548.

[5] J. Sorce and R.M. Wald, Gedanken experiments to destroy a black hole. II. Kerr-Newman black holes cannot be overcharged or overspun, Phys. Rev. D 96 (2017) 104014 [arXiv: 1707.05862] [INSPIRE].

[6] J.V. Rocha and V. Cardoso, Gravitational perturbation of the BTZ black hole induced by test particles and weak cosmic censorship in AdS spacetime, Phys. Rev. D 83 (2011) 104037 [arXiv: 1102.4352] [INSPIRE].

[7] S. Shaymatov, N. Dadhich, B. Ahmedov and M. Jamil, Five dimensional charged rotating minimally gauged supergravity black hole cannot be over-spun and/or over-charged in non-linear accretion, Eur. Phys. J. C 80 (2020) 481 [arXiv:1908.01195] [INSPIRE].

[8] K. Düztaş, M. Jamil, S. Shaymatov and B. Ahmedov, Testing cosmic censorship conjecture for extremal and near-extremal $(2+1)$-dimensional MTZ black holes, Class. Quant. Grav. $\mathbf{3 7}$ (2020) 175005 [arXiv: 1808.04711] [INSPIRE].

[9] S. Shaymatov, Magnetized Reissner-Nordström black hole restores cosmic censorship conjecture, Int. J. Mod. Phys. Conf. Ser. 49 (2019) 1960020 [INSPIRE].

[10] J. Jiang, B. Deng and Z. Chen, Static charged dilaton black hole cannot be overcharged by gedanken experiments, Phys. Rev. D 100 (2019) 066024 [arXiv:1909.02219] [INSPIRE].

[11] B. Ge, Y. Mo, S. Zhao and J. Zheng, Higher-dimensional charged black holes cannot be over-charged by gedanken experiments, Phys. Lett. B $\mathbf{7 8 3}$ (2018) 440 [arXiv:1712.07342] [INSPIRE].

[12] P. Wang, H. Wu and S. Ying, Validity of Thermodynamic Laws and Weak Cosmic Censorship for AdS Black Holes and Black Holes in a Cavity, arXiv:2002.12233 [INSPIRE].

[13] S. Ying, Thermodynamics and Weak Cosmic Censorship Conjecture of $4 D$ Gauss-Bonnet-Maxwell Black Holes via Charged Particle Absorption, Chin. Phys. C 44 (2020) 125101 [arXiv:2004.09480] [INSPIRE]. 
[14] B. Chen, F.-L. Lin and B. Ning, Gedanken Experiments to Destroy a BTZ Black Hole, Phys. Rev. D 100 (2019) 044043 [arXiv: 1902.00949] [InSPIRE].

[15] B. Gwak, Weak Cosmic Censorship Conjecture in Kerr-(Anti-)de Sitter Black Hole with Scalar Field, JHEP 09 (2018) 081 [arXiv:1807.10630] [InSPIRE].

[16] S.-J. Yang, J. Chen, J.-J. Wan, S.-W. Wei and Y.-X. Liu, Weak cosmic censorship conjecture for a Kerr-Taub-NUT black hole with a test scalar field and particle, Phys. Rev. D 101 (2020) 064048 [arXiv:2001.03106] [INSPIRE].

[17] S.-J. Yang, J.-J. Wan, J. Chen, J. Yang and Y.-Q. Wang, Weak cosmic censorship conjecture for the novel $4 D$ charged Einstein-Gauss-Bonnet black hole with test scalar field and particle, Eur. Phys. J. C 80 (2020) 937 [arXiv:2004.07934] [inSPIRE].

[18] D. Chen, Weak cosmic censorship conjecture in BTZ black holes with scalar fields, Chin. Phys. C 44 (2020) 015101 [arXiv: 1812.03459] [InSPIRE].

[19] B. Liang, S.-W. Wei and Y.-X. Liu, Weak cosmic censorship conjecture in Kerr black holes of modified gravity, Mod. Phys. Lett. A 34 (2019) 1950037 [arXiv: 1804.06966] [INSPIRE].

[20] J. Natario, L. Queimada and R. Vicente, Test fields cannot destroy extremal black holes, Class. Quant. Grav. 33 (2016) 175002 [arXiv:1601.06809] [INSPIRE].

[21] S. Hod, Weak Cosmic Censorship: As Strong as Ever, Phys. Rev. Lett. 100 (2008) 121101 [arXiv:0805.3873] [INSPIRE].

[22] V.E. Hubeny, Overcharging a black hole and cosmic censorship, Phys. Rev. D 59 (1999) 064013 [gr-qc/9808043] [INSPIRE].

[23] T. Jacobson and T.P. Sotiriou, Over-spinning a black hole with a test body, Phys. Rev. Lett. 103 (2009) 141101 [Erratum ibid. 103 (2009) 209903] [arXiv:0907.4146] [INSPIRE].

[24] G.E.A. Matsas and A.R.R. da Silva, Overspinning a nearly extreme charged black hole via a quantum tunneling process, Phys. Rev. Lett. 99 (2007) 181301 [arXiv:0706.3198] [INSPIRE].

[25] G.E.A. Matsas, M. Richartz, A. Saa, A.R.R. da Silva and D.A.T. Vanzella, Can quantum mechanics fool the cosmic censor?, Phys. Rev. D 79 (2009) 101502 [arXiv:0905.1077] [INSPIRE].

[26] M. Richartz and A. Saa, Challenging the weak cosmic censorship conjecture with charged quantum particles, Phys. Rev. D 84 (2011) 104021 [arXiv:1109.3364] [INSPIRE].

[27] R. Gregory and R. Laflamme, Black strings and p-branes are unstable, Phys. Rev. Lett. 70 (1993) 2837 [hep-th/9301052] [INSPIRE].

[28] L. Lehner and F. Pretorius, Black Strings, Low Viscosity Fluids, and Violation of Cosmic Censorship, Phys. Rev. Lett. 105 (2010) 101102 [arXiv:1006.5960] [INSPIRE].

[29] P. Figueras, M. Kunesch and S. Tunyasuvunakool, End Point of Black Ring Instabilities and the Weak Cosmic Censorship Conjecture, Phys. Rev. Lett. 116 (2016) 071102 [arXiv: 1512.04532] [INSPIRE].

[30] P. Figueras, M. Kunesch, L. Lehner and S. Tunyasuvunakool, End Point of the Ultraspinning Instability and Violation of Cosmic Censorship, Phys. Rev. Lett. 118 (2017) 151103 [arXiv: 1702.01755] [INSPIRE].

[31] V.E. Hubeny and M. Rangamani, Unstable horizons, JHEP 05 (2002) 027 [hep-th/0202189] [INSPIRE]. 
[32] J.E. Santos and B. Way, Neutral Black Rings in Five Dimensions are Unstable, Phys. Rev. Lett. 114 (2015) 221101 [arXiv: 1503.00721] [INSPIRE].

[33] G.T. Horowitz, J.E. Santos and B. Way, Evidence for an Electrifying Violation of Cosmic Censorship, Class. Quant. Grav. 33 (2016) 195007 [arXiv:1604.06465] [INSPIRE].

[34] T. Crisford and J.E. Santos, Violating the Weak Cosmic Censorship Conjecture in Four-Dimensional Anti-de Sitter Space, Phys. Rev. Lett. 118 (2017) 181101 [arXiv: 1702.05490] [INSPIRE].

[35] T. Crisford, G.T. Horowitz and J.E. Santos, Attempts at vacuum counterexamples to cosmic censorship in AdS, JHEP 02 (2019) 092 [arXiv: 1805.06469] [INSPIRE].

[36] T. Crisford, G.T. Horowitz and J.E. Santos, Testing the Weak Gravity-Cosmic Censorship Connection, Phys. Rev. D 97 (2018) 066005 [arXiv:1709.07880] [INSPIRE].

[37] N. Arkani-Hamed, L. Motl, A. Nicolis and C. Vafa, The String landscape, black holes and gravity as the weakest force, JHEP 06 (2007) 060 [hep-th/0601001] [INSPIRE].

[38] E. Palti, The Weak Gravity Conjecture and Scalar Fields, JHEP 08 (2017) 034 [arXiv: 1705. 04328] [INSPIRE].

[39] E. Palti, The Swampland: Introduction and Review, Fortsch. Phys. 67 (2019) 1900037 [arXiv: 1903.06239] [INSPIRE].

[40] P. Saraswat, Weak gravity conjecture and effective field theory, Phys. Rev. D 95 (2017) 025013 [arXiv: 1608.06951] [INSPIRE].

[41] S.A. Hartnoll, C.P. Herzog and G.T. Horowitz, Building a Holographic Superconductor, Phys. Rev. Lett. 101 (2008) 031601 [arXiv:0803.3295] [INSPIRE].

[42] R.-G. Cai, L. Li, L.-F. Li and R.-Q. Yang, Introduction to Holographic Superconductor Models, Sci. China Phys. Mech. Astron. 58 (2015) 060401 [arXiv:1502.00437] [INSPIRE].

[43] S.A. Hartnoll, C.P. Herzog and G.T. Horowitz, Holographic Superconductors, JHEP 12 (2008) 015 [arXiv: 0810.1563] [INSPIRE].

[44] G.T. Horowitz and J.E. Santos, Further evidence for the weak gravity-cosmic censorship connection, JHEP 06 (2019) 122 [arXiv:1901.11096] [INSPIRE].

[45] T.-T. Hu, Y. Song, S. Sun, H.-B. Li and Y.-Q. Wang, Weak cosmic censorship in Born-Infeld electrodynamics and bound on charge-to-mass ratio, Eur. Phys. J. C 80 (2020) 147 [arXiv: 1906.00235] [INSPIRE].

[46] C.A.R. Herdeiro, E. Radu and H. Rúnarsson, Kerr black holes with self-interacting scalar hair: hairier but not heavier, Phys. Rev. D 92 (2015) 084059 [arXiv: 1509.02923] [InSPIRE].

[47] C.A.R. Herdeiro and E. Radu, Spherical electro-vacuum black holes with resonant, scalar Q-hair, Eur. Phys. J. C 80 (2020) 390 [arXiv:2004.00336] [inSPIRE].

[48] H.-B. Li, Y.-B. Zeng, Y. Song and Y.-Q. Wang, Self-interacting multistate boson stars, arXiv:2006.11281 [INSPIRE].

[49] C.A.R. Herdeiro, E. Radu and H.F. Rúnarsson, Spinning boson stars and Kerr black holes with scalar hair: the effect of self-interactions, Int. J. Mod. Phys. D 25 (2016) 1641014 [arXiv: 1604.06202] [INSPIRE].

[50] S. Valdez-Alvarado, R. Becerril and L.A. Ureña-López, Fermion-boson stars with a quartic self-interaction in the boson sector, Phys. Rev. D 102 (2020) 064038 [arXiv:2001.11009] [INSPIRE]. 
[51] F.E. Schunck and D.F. Torres, Boson stars with generic selfinteractions, Int. J. Mod. Phys. D 9 (2000) 601 [gr-qc/9911038] [INSPIRE].

[52] F.E. Schunck and E.W. Mielke, General relativistic boson stars, Class. Quant. Grav. 20 (2003) R301 [arXiv:0801.0307] [INSPIRE].

[53] P. Breitenlohner and D.Z. Freedman, Positive Energy in anti-de Sitter Backgrounds and Gauged Extended Supergravity, Phys. Lett. B 115 (1982) 197 [InSPIRE].

[54] L. Mezincescu and P.K. Townsend, Stability at a Local Maximum in Higher Dimensional Anti-de Sitter Space and Applications to Supergravity, Annals Phys. 160 (1985) 406 [INSPIRE].

[55] P. Breitenlohner and D.Z. Freedman, Stability in Gauged Extended Supergravity, Annals Phys. 144 (1982) 249 [INSPIRE].

[56] P.K. Townsend, Positive Energy and the Scalar Potential in Higher Dimensional (Super)gravity Theories, Phys. Lett. B 148 (1984) 55 [inSPIRE].

[57] G.T. Horowitz, N. Iqbal, J.E. Santos and B. Way, Hovering Black Holes from Charged Defects, Class. Quant. Grav. 32 (2015) 105001 [arXiv: 1412.1830] [InSPIRE].

[58] M. Headrick, S. Kitchen and T. Wiseman, A New approach to static numerical relativity, and its application to Kaluza-Klein black holes, Class. Quant. Grav. 27 (2010) 035002 [arXiv: 0905. 1822] [INSPIRE].

[59] O.J.C. Dias, J.E. Santos and B. Way, Numerical Methods for Finding Stationary Gravitational Solutions, Class. Quant. Grav. 33 (2016) 133001 [arXiv:1510.02804] [INSPIRE]. 